\title{
Novel bioabsorbable interbody fusion spacer-assisted fusion for correction of spinal deformity
}

\author{
Rebekah C. Austin, M.D., Charles L. Branch, JR., M.D., \\ AND JOSEPH T. AleXANDER, M.D. \\ Department of Neurosurgery, Wake Forest University Medical Center, Winston-Salem, North \\ Carolina
}

\begin{abstract}
Object. The authors report the cases of 12 patients with medically refractory mechanical low-back pain and intermittent radicular symptoms in whom radiography demonstrated evidence of multilevel lumbosacral degenerative kyphotic and scoliotic deformity and spondylolisthesis.

Methods. These patients underwent multilevel posterior lumbar interbody fusion in which Macropore bioabsorbable spacers were placed. Each patient underwent at least 1 year of clinical and radiographic follow up.

Conclusions. This series illustrates the novel use of bioabsorbable interbody spacers and fusion technique for correction of spinal deformity due to advanced degenerative kyphoscoliosis and spondylolisthesis.
\end{abstract}

\section{KEY WORDS • spinal deformity - posterior lumbar interbody fusion • bioabsorbable implant}

Degenerative spinal deformity is a formidable problem in the aging population. With advancing age, disc dessication and degeneration occur as do osteophyte formation and gradual reduction in the volume of cancellous bone throughout the spinal column. ${ }^{8}$ This process may be significantly accelerated in postmenopausal women, smokers, and those with a congenital predisposition such as achondroplasia. When these degenerative processes occur asymmetrically, kyphosis and scoliosis may contribute to a rapidly narrowing lumbar canal. The clinical syndrome associated with these findings is chronic low-back pain that worsens over time and may be exacerbated by standing, walking, or back extension. Radiculopathy or dysesthesias may be present but generally are less prominent features of the pain syndrome. ${ }^{8}$

In the treatment of these deformities, decompressive laminectomy with or without facetectomy and discectomy may provide adequate neural decompression; however, this procedure fails to correct, and may worsen, a developing degenerative spinal deformity. ${ }^{8}$ In these instances recurrent pain manifests and revision surgery is often indi-

Abbreviations used in this paper: $\mathrm{AP}=$ anteroposterior; $\mathrm{CT}=$ computerized tomography; PLIF = posterior lumbar interbody fusion. cated. The PLIF technique provides anterior structural support and foraminal distraction by means of an interbody graft as well as posterior immobilization by pedicle screw/rod- or plate-augmented fixation. ${ }^{1,10}$ Intertransverse onlay autograft is used to augment posterior fusion. This technique allows for the correction of degenerative kyphosis and scoliosis in addition to neural decompression. Excellent fusion rates have been well documented. ${ }^{1,10}$

Interbody grafts used in PLIF have historically included autograft, cadaveric allograft, titanium and stainless steel implants, and most recently custom devices made of bioabsorbable polymers including polyhydroxy acids. Macropore (Macropore Biosurgery, Inc., San Diego, CA) is a polyhydroxy acid polymer that may prove to be suitable for placement as an interbody graft. It has been approved by the Food and Drug Administration for use as a cement restrictor and has been used in this study in an investigational capacity. Advantages of bioabsorbable materials include biocompatibility with neural tissue, predictable degradation, lack of neuroimaging-related exuberant inflammatory reaction, and lack of neuroimagingrelated artifacts. In addition, there is no significant toxicity because the degradation products are typically carbon dioxide and water. Encouraging preliminary results have previously been reported with the use of Macropore spac- 
TABLE 1

Summary of surgical treatment and results*

\begin{tabular}{rrllll}
\hline \hline Case No. & Age (yrs), Sex & \multicolumn{1}{c}{ Deformity } & PLIF Segments & Result & Complication \\
\hline 1 & $1, \mathrm{~F}$ & scoliosis, spondy & L1-4 & fusion & no \\
2 & $77, \mathrm{~F}$ & kyphoscoliosis & L2-5 & fusion & no \\
3 & $72, \mathrm{~F}$ & spondy & L4-S1 & fusion & no \\
4 & $64, \mathrm{M}$ & spondy & L4-S1 & fusion & no \\
5 & $61, \mathrm{~F}$ & spondy & L3-S1 & fusion & no \\
6 & $70, \mathrm{~F}$ & kyphoscoliosis & L2-5 & fusion & no \\
7 & $77, \mathrm{~F}$ & scoliosis, spondy & L3-5 & fusion & no \\
8 & $58, \mathrm{~F}$ & scoliosis & L2-4 & fusion & no \\
9 & $50, \mathrm{M}$ & spondy & L4-S1 & fusion & no \\
10 & $77, \mathrm{~F}$ & scoliosis & L2-S1 & fusion & fusion \\
11 & $67, \mathrm{~F}$ & kyphoscoliosis & L4-S1 & fusion & no \\
12 & $71, \mathrm{M}$ & spondy & & & \\
\hline
\end{tabular}

* spondy = spondylolisthesis.

ers in PLIF constructs. Further investigation is warranted to determine fusion rates, graft durability, and patient selection criteria. We present a small series of 12 cases in which bioabsorbable interbody graft-assisted PLIF resulted in correction of degenerative spinal deformity; patients attended more than 1 year of clinical and radiographic follow up.

\section{CLINICAL MATERIAL AND METHODS}

\section{Patient Population}

We treated 12 patients with spinal deformity. Patients ranged in age from 1 to 77 years. There were nine female and three male patients.

\section{Surgical Technique}

Each patient underwent bilateral foraminotomies and partial facetectomies for decompression of spinal stenosis and multilevel PLIF involving pedicle screw internal fixation for correction of degenerative kyphotic scoliotic deformity and/or spondylolisthesis. Interbody fusion was performed by placing the Macropore bioabsorbable spacers bilaterally at each level; the implants were filled with locally harvested autograft, and autograft was placed over the transverse processes as well. Perioperative antibiotic and steroid agents were administered. Patients were out of bed and ambulatory on postoperative Day 1 while wearing a lumbosacral corset.

\section{RESULTS}

Surgery was successful in all 12 patients in the treatment of scoliotic, kyphoscoliotic, and spondylolisthetic deformities. A summary of the PLIF-treated segments and fusion results is presented in Table 1. Delayed wound healing was documented in one case. Pseudarthrosis and adjacent-level stenosis were absent in all cases. No new neurological deficits developed.

\section{ILLUSTRATIVE CASES}

\section{Case 2}

This 77-year-old woman with a medical history of noninsulin dependent diabetes mellitus and hypertension was referred for longstanding low-back pain and intermittent right-sided lower-extremity pain radiating to her buttock and hip. The pain continued to worsen despite conservative therapy and was exacerbated by walking or standing whereas it was partially relieved by rest or sedentary posi-

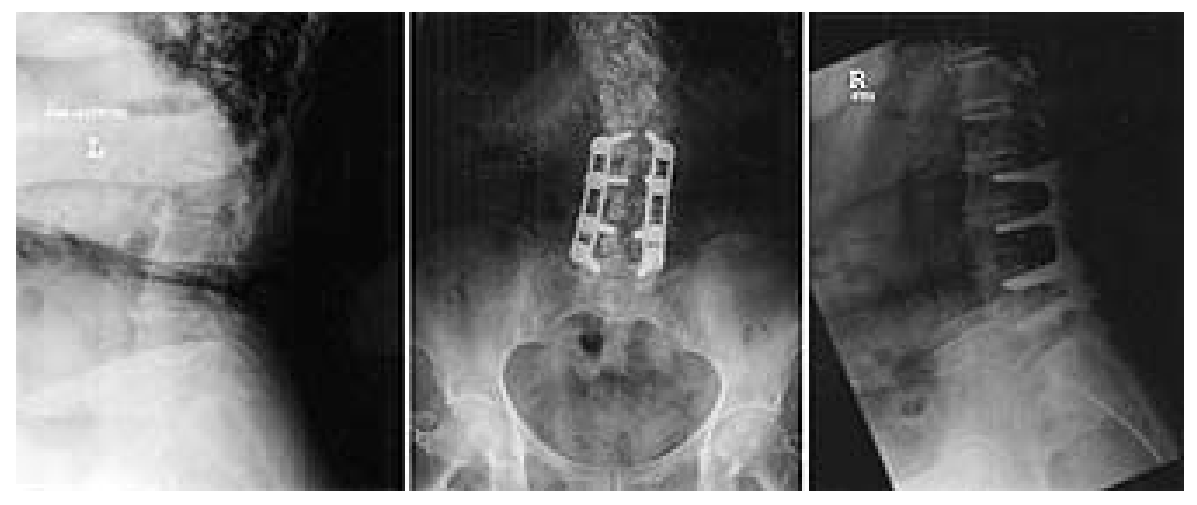

Fig. 1. Case 2. Left: Preoperative lateral radiograph revealing multilevel degenerative spondylotic disease, loss of lordosis, and osteopenia (AP view unavailable). Center and Right: Postoperative AP and lateral radiographs demonstrating instrumentation and improved alignment. 
tion. Physical examination revealed excellent strength in isolated muscle groups, negative straight leg raise test, and normal tandem gait. Paraspinous muscle spasms and palpable lumbar scoliosis were present. Lumbar CT myelography revealed severe degenerative disc disease throughout the spine with marked L2-S1 canal stenosis secondary to disc and osteophytic protrusion, facet hypertrophy, and kyphoscoliotic deformity. Plain AP and lateral radiography confirmed the CT findings (Fig. 1 left) as well as advanced osteopenia. Magnetic resonance imaging could not be performed because of an indwelling cardiac pacemaker. The patient underwent L2-5 decompression and PLIF. She was discharged on postoperative Day 6 in stable condition. She continued to undergo muscle relaxer and oral narcotic pain therapies for 8 weeks. At 1 year, she was clinically improved, and AP and lateral radiography revealed a stable fusion construct with improved alignment (Fig. 1 center and right). A lumbar three-dimensional CT reconstruction was obtained to evaluate the interbody graft and spinal alignment in the immediate postoperative period (Fig. 2 upper) and at 9-month follow-up examination (Fig. 2 lower).

\section{Case 6}

This 70-year-old woman with a medical history significant for subarachnoid hemorrhage after undergoing a craniotomy for multiple aneurysm clippings 8 years prior and atrial fibrillation presented with incapacitating low-back pain that radiated bilaterally to the posterior thighs. There was a significant mechanical component to her pain syndrome, which continued to worsen despite years of conservative therapy. Physical examination was notable only for palpable lumbar scoliosis. Thoracolumbar CT my-

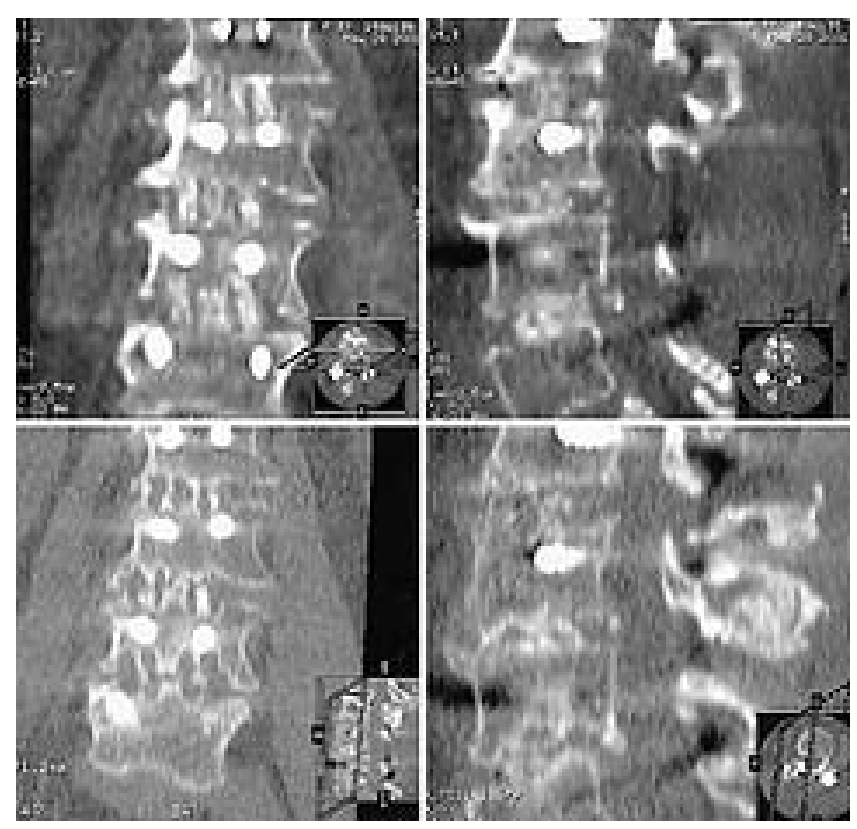

Fig. 2. Case 2. Postoperative three-dimensional CT reconstructions. Upper Left and Right: Immediate postoperative images. Lower Left and Right: Nine-month images. Both sets reveal maintained deformity correction and evolution of osseous fusion.

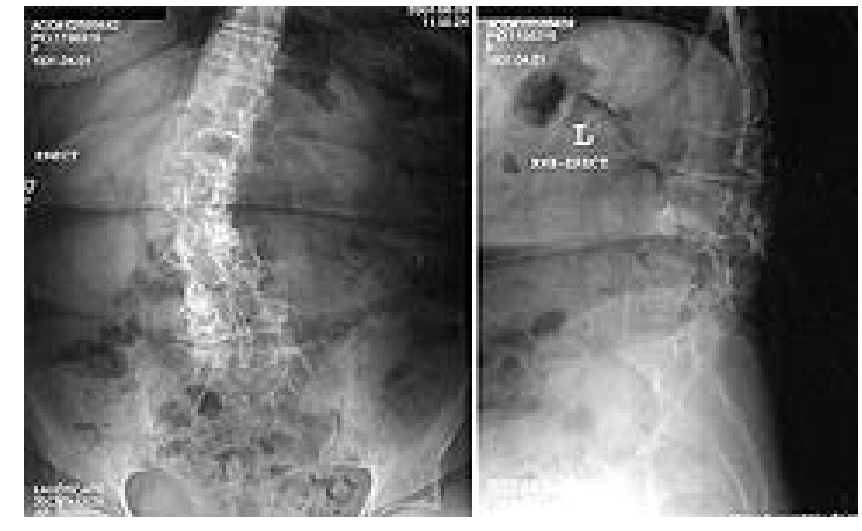

Fig. 3. Case 6. Preoperative AP and lateral radiographs.

elography revealed severe lumbar degenerative disc disease and scoliosis with marked stenosis secondary to deformity, as well as osteophyte and facet hypertrophy. Lateral and AP radiography confirmed these findings as well as advanced osteopenia (Fig. 3). The patient underwent L2-5 decompression and PLIF and T10-L5 pedicle screw/rod fixation. Immediate postoperative imaging demonstrated correction of scoliotic deformity with disc height restoration at the treated levels (Fig. 4). The patient received inpatient physical therapy to assist her with early mobilization and was discharged on postoperative Day 8 after an uneventful hospitalization. At 1-year follow-up examination, she was receiving nonsteroidal antiinflammatory drugs for intermittent back pain and had resumed her desired activity level. Lateral and AP radiography demonstrated maintained deformity correction and excellent fusion (Fig. 5).

\section{Case 8}

This 58-year-old woman with a medical history significant for fibromyalgia, hypertension, and childhood polio was referred because of longstanding episodic low-back pain that had worsened in frequency and severity within the past 4 to 6 weeks. She complained of severe back pain with left lower-extremity radicular pain, which worsened while standing and was only minimally relieved by rest. Physical examination was significant only for paraspinous

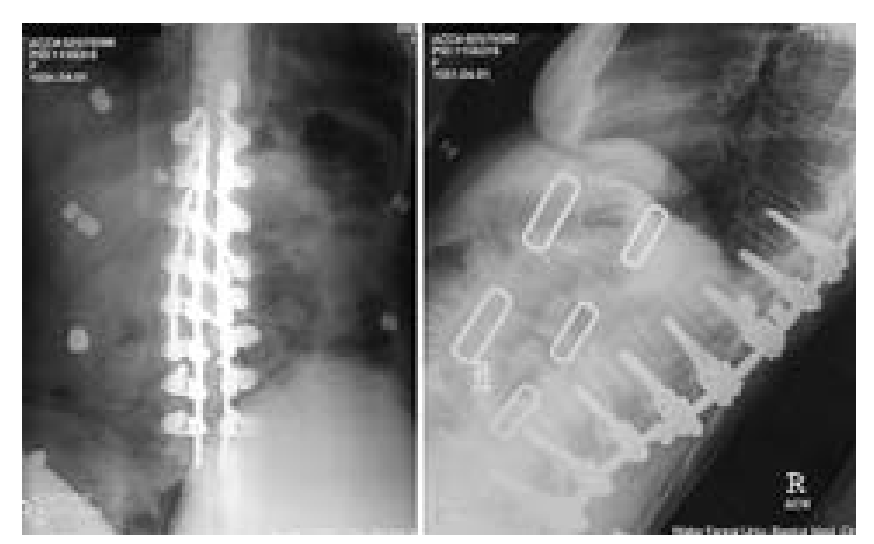

Fig. 4. Case 6. Postoperative AP and lateral radiographs. 
muscle tenderness and spasm. Lumbar magnetic resonance imaging revealed degenerative collapse and obliteration of L2-3 and L3-4 disc spaces with multilevel disc disease and stenosis secondary to buckled ligamentum flavum and facet hypertrophy. Lateral and AP radiography again demonstrated loss of L2-3 and L3-4 disc height associated with degenerative scoliotic deformity at these levels (Fig. 6 upper and lower left). The patient underwent L2-4 decompression and PLIF as well as L2-4 pedicle screw/rod fixation. The patient experienced only incisionrelated pain and was discharged on postoperative Day 5. Immediate postoperative imaging confirmed excellent correction of scoliosis and restoration of disc height at the treated levels (Fig. 6 upper and lower right). These findings were present radiographically 1 year postoperatively (Fig. 7). These imaging studies also demonstrated solid fusion and partial resorption of the interbody spacers. The patient has resumed her previous activities and experiences only occasional back pain which she treats with nonsteroidal antiinflammatory drugs.

\section{Case 11}

This 68-year-old woman with a medical history significant for osteoporosis and mitral valve prolapse presented for evaluation of her severe low-back pain, which occasionally radiated to her right hip and groin, and intermittent, right-sided lower-extremity paresthesias. Lumbar magnetic resonance imaging demonstrated multilevel degenerative disc disease with severe L2-S1 stenosis secondary to disc protrusion, facet hypertrophy, and scoliotic deformity. Lateral and AP radiography revealed marked lumbar scoliosis and advanced osteopenia (Fig. 8 upper and lower). The patient underwent L2-S1 and PLIF for realignment. We also performed L2-S1 pedicle screw/rod fixation. Immediate postoperative radiography confirmed restoration of lordosis and correction of lateral scoliosis (Fig. 8 upper and lower right). The patient was discharged on postoperative Day 7 after an uneventful hospital course. At 1-year follow-up examination, she complained

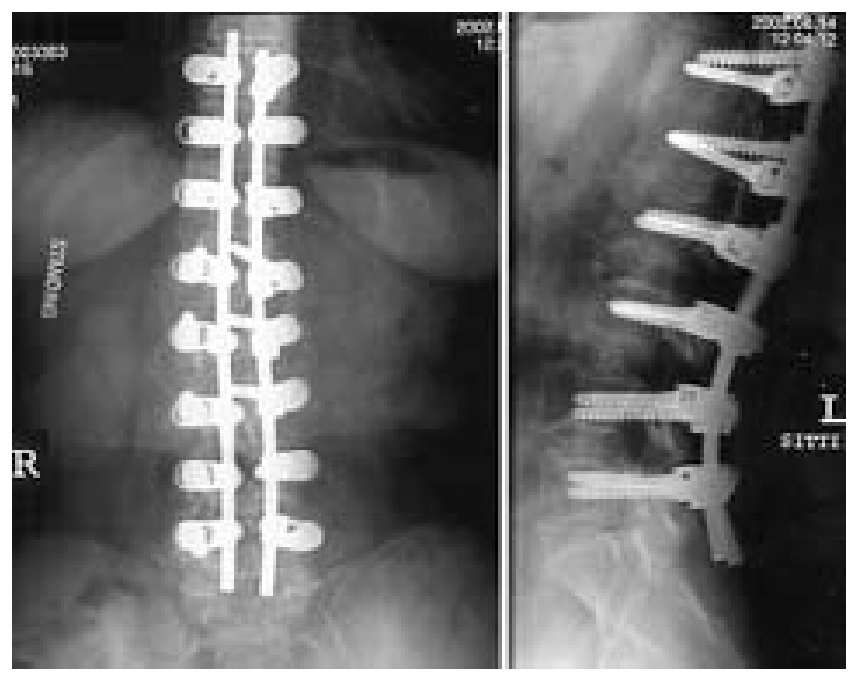

Fig. 5. Case 6. One-year follow-up AP (left) and lateral (right) radiographs.

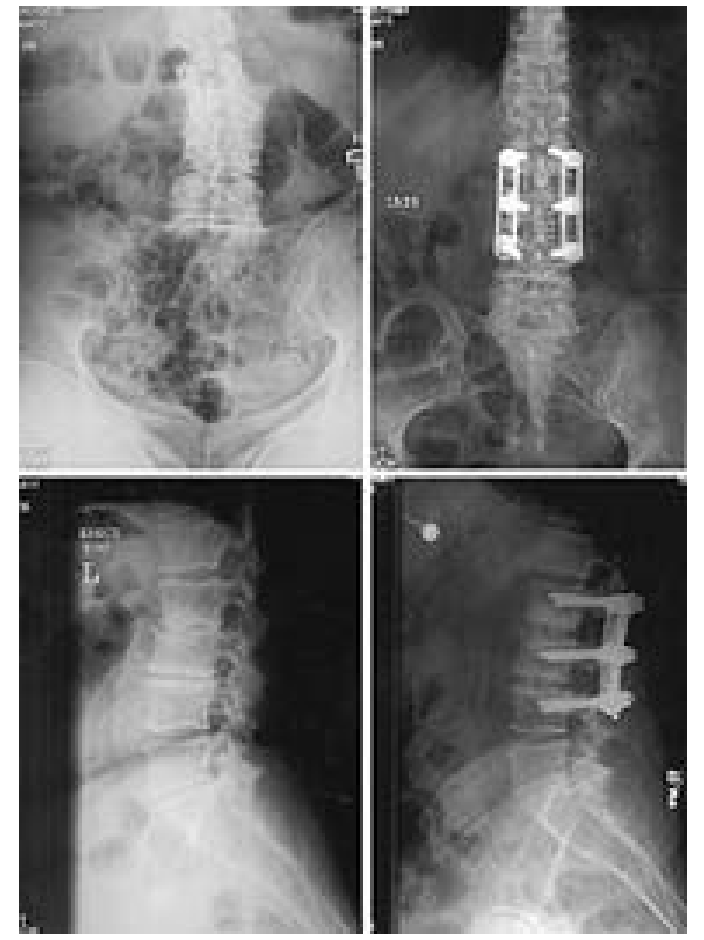

Fig. 6. Case 8. Preoperative (upper left) and postoperative (upper right) AP radiographs. Preoperative (lower left) and postoperative (lower right) lateral radiographs.

only of occasional hip pain. Radiography demonstrated excellent fusion and maintenance of alignment (Fig. 9).

\section{DISCUSSION}

Ralph Cloward, was one of the first surgeons to recognize and apply the advantages of interbody fusion techniques in the treatment of lumbar degenerative disease in the 1940s. By using autograft- or allograft-assisted PLIF, he was able to achieve consistent successful fusion rates., ${ }^{2,3}$ The carpentry involved in this procedure was technically demanding and therefore never widely adopted. With the

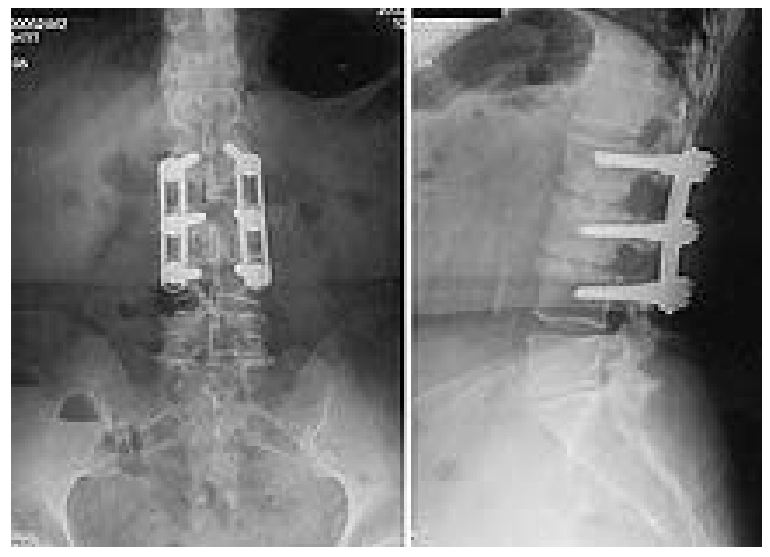

Fig. 7. Case 8. One-year follow-up AP (left) and lateral (right) radiographs. 


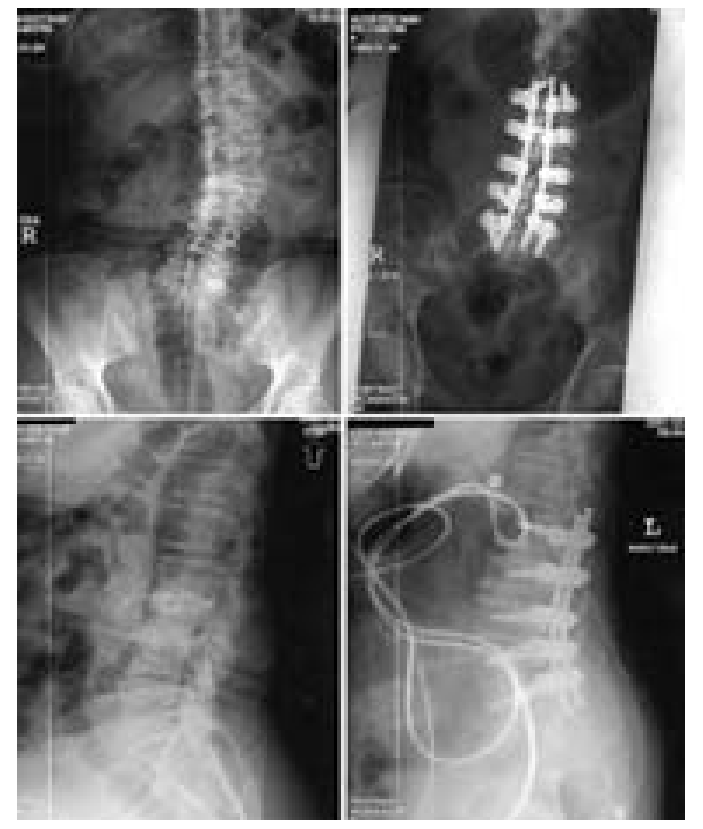

Fig. 8. Case 11. Preoperative (upper left) and postoperative (upper right) AP radiographs. Preoperative (lower left) and postoperative (lower right) lateral radiographs.

introduction of posterior instrumentation systems, Cloward's technique was largely abandoned. In the 1990s, because of better understanding of spinal biomechanics, growing dissatisfaction with both clinical and radiographic outcomes after posterior intertransverse fusion in the treatment of degenerative spondylosis ${ }^{7}$ and the regulatory status of the pedicle screw, interest in the interbody fusion was renewed.

Initial interbody grafts included auto- and allograft bone, which was fashioned by the surgeon prior to insertion. This time-consuming task spurred the search for more reproducible alternatives. Successful outcomes were obtained in preclinical studies involving stand-alone titanium cylindrical cages in PLIF, and this led to Food and Drug Administration approval and widespread application of this technique. ${ }^{5,9}$ In some cases, because strict patient selection and careful adherence to recommended insertion protocols were not followed, an unexpectedly high construct failure and revision rate resulted. Because these devices were composed of titanium, revision and removal were challenging, and the assessment of the fusion progress was impeded. In response to these complications, the traditional technique of allograft-augmented PLIF has been modified to include preformed cortical allograft bone grafts and specialized instrumentation systems that facilitate safe and precise insertion. ${ }^{1}$ These grafts are supplemented with segmental fixation devices and onlay and interbody autograft; high fusion rates have been consistently achieved. ${ }^{1,10}$ Limited allograft supply and potential for transmission of infectious disease continue to affect the application of this technique in the US.

Posterior lumbar fusion involving placement of interbody devices filled with locally harvested autograft and augmented by pedicle screw/rod fixation provides an excellent tool for realignment of the degenerative kyphosco-

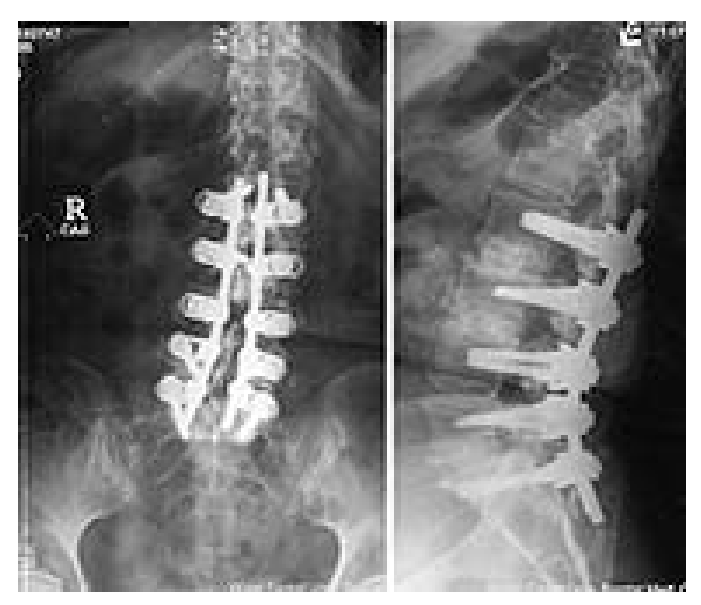

Fig. 9. Case 11. One-year follow-up AP (left) lateral (right) radiographs.

liotic and spondylotic lumbar spine. Generous laminotomies and partial or complete facetectomies provide adequate neural decompression and facilitate manipulation of the osseous elements. In addition to providing a medium for osteoconduction and anterior load sharing, an interbody graft restores disc height and aids in reestablishing lumbar lordosis. Once the interbody spacers and autograft are inserted, pedicle screws and rods can be placed with compression at appropriate levels to provide the necessary rigidity to maintain the restored alignment.

In spinal applications, bioresorbable materials may only serve as interbody spacers and fixation devices. Once the support or "tension-band" function has been fulfilled and the fusion has matured, the hardware has no further function and, because of its associated artifacts, hinders imaging studies. The biomechanical demands on an interbody spacer are considerably less than those of a stand-alone fixation screw/plate construct. ${ }^{6}$ The gradual degradation of an interbody graft may promote arthrodesis by exposing the maturing interbody bone to increased load sharing. This mechanism is in contrast to the stress riser at a screw-rod junction, which may significantly alter the expected local degradation rate of the biopolymer and lead to sudden, catastrophic failure of this type of implant. ${ }^{4}$

The versatile nature of bioresorbable material allows it to be formed into cages, dowels, and interbody spacer shapes. In addition, its desirable strength and degradation characteristics, lack of imaging-related artifact, low potential for foreign-body reaction, and the biocompatibility with the dura and neural tissue make it a promising material for this application. ${ }^{4}$ Patients with advanced spinal degenerative deformity often require multilevel fusions involving instrumentation for realignment, which increases the likelihood of pseudarthrosis and makes imaging evaluation crucial in their subsequent management. The radiolucent feature of Macropore interbody spacers allows for earlier radiographic evidence of fusion and deformity correction without the artifact generated by titanium cages or bone dowels. In the cases presented here successful clinical and radiographic results were demonstrated 12 to 18 months following surgery for degenerative spinal deformity correction. The authors recommend the continued consideration of this technique. 


\section{References}

1. Barnes B, McLaughlin M, Rodts G, et al: PLIF and impacted PLIF, in Resnick DK, Haid RW Jr, (eds): Surgical Management of Low Back Pain. Rolling Meadows, IL: American Association of Neurological Surgeons, 2001, pp 93-102

2. Cloward R: The treatment of ruptured lumbar intervertebral discs by vertebral body fusion III: method of use of banked bone. Ann Surg 136:987-992, 1952

3. Cloward RB: The treatment of ruptured lumbar intervertebral discs by vertebral body fusion I: indications, operative technique, aftercare. J Neurosurg 10:154-168, 1953

4. Gogolewski S: Bioresorbable polymers in trauma and bone surgery. Injury 31 (Suppl 4): 28-32, 2000

5. Kuslich SD, Ulstrom LL, Griffith SL, et al: The Bagby and Kuslich method of lumbar interbody fusion. History, techniques, and 2-year follow-up results in the United States prospective, multicenter trial. Spine 23:1267-1278, 1998

6. Lastra JJ, Willis BH, Benzel EC: Geometrical considerations for the surgical management of low back pain, in Resnick DK, Haid RW Jr, (eds): Surgical Management of Low Back Pain. Rolling Meadows, IL: American Association of Neurological Surgeons, 2001, pp 47-60

7. Nguyen PH, Trost GR: Posterolateral fusion in the management of low back pain, in Resnick DK, Haid RW Jr, (eds): Surgical Management of Low Back Pain. Rolling Meadows, IL: American Association of Neurological Surgeons, 2001, pp 69-82

8. Pappas CTE, Sonntag VKH: Degenerative disorders of the spine: lumbar stenosis, in Menezes AH, Sonntag VKH, (eds): Principles of Spinal Surgery. New York: McGraw-Hill, 1996, Vol 1, pp 631-644

9. Ray CD: Threaded titanium cages for lumbar interbody fusions. Spine 22:667-680, 1997

10. Sasso RC, Reilly TM: Anterior lumbar interbody fusion: threaded bone dowels versus titanium cages, in Resnick DK, Haid RW Jr, (eds): Surgical Management of Low Back Pain. Rolling Meadows, IL: American Association of Neurological Surgeons, 2001, pp 103-116

Manuscript received November 21, 2002.

Accepted in final form December 23, 2002.

Address reprint requests to: Charles L. Branch, Jr. M.D., Department of Neurosurgery, Wake Forest University Medical Center, Medical Center Boulevard, Winston-Salem, North Carolina 27105. email: cbranch@wfubmc.edu. 